\title{
FIBRODYSPLASIA OSSIFICANS PROGRESSIVA: RARE AND LIMITING DISEASE
}

Henrique Perez Filik',*, Tatiana Freitas Tourinho ${ }^{1}$, Maria Lucia Lemos Lopes ${ }^{1}$, Maria Odete Esteves Hilario ${ }^{1}$, Eduardo Rosa de Oliveira $^{1}$, Thiago Willers ${ }^{1}$, Rafael Coradin ${ }^{1}$, Raissa Velasques de Figueiredo ${ }^{1}$, Bruno Trevisan ${ }^{1}$, Gabriela Sasso Padilha ${ }^{1}$, Luana Ribeiro Carlos ${ }^{1}$

1. Universidade Federal de Ciências da Saúde de Porto Alegre, Porto Alegre (RS), Brazil.

*Corresponding author: gilberto_scanagatta@hotmail.com

\section{BACKGROUND}

Fibrodysplasia ossificans progressiva (FOP) or myositis ossificans is a rare connective tissue and autosomal dominant disease, with variable expression. Its pathophysiology is not yet known. Repetitive traumas can trigger the process. It starts in childhood and in young adults with a predilection for males. The involvement is progressive, with loss of mobility, and consequent joint deformation. The initial manifestation is characterized by soft tissue edema, due to inflammatory processes, resulting in bone neoformation in muscles, tendons, fasciae, ligaments and joint capsules, which are calcified. Repetitive trauma is the most common causal factor of the disease (corresponding to $60-75 \%$ of cases), and it can also be attributed to hereditary factors in a minority. The most affected places are the thighs and buttocks. Mineralization is seen on the radiograph approximately two to four weeks after the start of the process. We report the case of a patient with difficulty walking as a result of calcifications after an infectious condition.

\section{CASE REPORT}

A 35-year-old white female patient developed polyarthralgia and arthritis in her left knee after encephalitis in 2006. Knee and hip radiographs were taken with the presence of periarticular calcifications. Subsequently, scintigraphy was performed, which demonstrated hyper-uptake in the clavicle, bilateral hip joint, ischium, iliac and bilateral femur. Pulse therapy with methylprednisolone was performed with good response to acute encephalitis and improvement of arthritis. She has an important limitation to perform hip movements, she has valgus knees with limited movement on physical examination. Radiographs with massive calcification adjacent to the bilateral hip joint and knees, changes in radiological aspect compatible with ossifying myositis. Laboratory tests of normal bone metabolism. Progressively maintains knee and hip arthralgia with restricted hip abduction, gait difficulty.

\section{CONCLUSION}

Fibrodysplasia ossificans progressiva should be promptly identified based on clinical history, physical examination and radiographic findings with the presence of ossification in soft tissues. The differential diagnosis is made with Albright's hereditary osteodystrophy, pseudomalignant heterotopic calcification, progressive bone heteroplasia and osteosarcoma. Numerous treatments have been used with low effectiveness in controlling the disease. Indications for surgical intervention include persistence of pain, muscle weakness and limited range of motion. Recurrence can occur spontaneously or precipitated by invasive procedures such as injections, biopsies, punctures, which can trigger or accelerate the inflammatory process and ectopic calcifications. 